\title{
Analytical Devices Based on Direct Synthesis of DNA on Paper
}

\section{Citation}

Glavan, Ana C., Jia Niu, Zhen Chen, Firat Güder, Chao-Min Cheng, David Liu, and George M. Whitesides. 2016. "Analytical Devices Based on Direct Synthesis of DNA on Paper." Analytical Chemistry 88 (1) (January 5): 725-731. doi:10.1021/acs.analchem.5b02822.

\section{Published Version}

10.1021/acs.analchem.5b02822

\section{Permanent link}

http://nrs.harvard.edu/urn-3:HUL.InstRepos:33490484

\section{Terms of Use}

This article was downloaded from Harvard University's DASH repository, and is made available under the terms and conditions applicable to Open Access Policy Articles, as set forth at http:// nrs.harvard.edu/urn-3:HUL.InstRepos:dash.current.terms-of-use\#OAP

\section{Share Your Story}

The Harvard community has made this article openly available.

Please share how this access benefits you. Submit a story.

\section{Accessibility}




\section{Analytical Devices Based on Direct Synthesis of DNA on Paper}

Ana C. Glavan ${ }^{\dagger}$, Jia Niu ${ }^{\dagger}$, Zhen Chen, Firat Guder, Chao-Min Cheng, David Liu, "George M. Whitesides*

Department of Chemistry \& Chemical Biology, Harvard University, Cambridge, MA 02138, U.S.A.

*Corresponding authors

E-mail: gwhitesides@gmwgroup.harvard.edu liu@chemistry.harvard.edu

$\dagger$ Both authors contributed equally to this work.

Target Journal(s): Anal Chem 


\begin{abstract}
This paper addresses a growing need in clinical diagnostics for parallel, multiplex analysis of biomarkers from small biological samples. It describes a new procedure for assembling microarrays of ssDNA and proteins on paper. This method starts with the synthesis of DNA oligonucleotides covalently linked to paper, and proceeds to generate DNA arrays capable of simultaneously capturing DNA, DNA-conjugated protein antigens, and DNA-conjugated antibodies. The synthesis of ssDNA oligonucleotides on paper is convenient and effective, with $32 \%$ of the oligonucleotides cleaved and eluted from the paper substrate being full-length by HPLC for a 32-mer. These ssDNA arrays can be used to detect fluorophore-linked DNA oligonucleotides in solution, and as the basis for DNA-directed assembly of microarrays of DNA-conjugated capture antibodies on paper, detect protein antigens by sandwich ELISAs. Paper-anchored ssDNA arrays with different sequences can be used to assemble paper-based devices capable of detecting DNA and antibodies in the same device, and enable simple microfluidic paperbased devices.
\end{abstract}




\section{Introduction}

Microarrays are convenient tools for the multiplex analysis of several biological samples in clinical diagnostics. ${ }^{[1,2]}$ A microarray is a solid support bearing microscopic features that can detect specific target molecules and generate diagnostic data. ${ }^{[3]}$ The standard method of fabrication for microarrays is pin-spotting - a method in which a robotic system deposits small volumes of a solution containing a probe (usually DNA, RNA, antibody, or protein) onto a glass, silicon, or polymer-based substrate. ${ }^{[4]}$ Alternative methods include microstamping, inkjet printing, laser writing, or electrospray deposition, among others ${ }^{[5,6]}$. These substrates can be derivatized with poly-L-lysine, polyamidoamine dendrimer, amino-terminated silanes, aldehydes, carboxylic acids, or other reactive groups that facilitate attachment. ${ }^{[7]}$ Existing methods for the fabrication of microarrays rely on complex equipment for processing, and require a series of lengthy purification and functionalization steps; the substrates commonly used are neither flexible nor inexpensive, and are difficult to integrate in low-cost diagnostics systems intended for use in resource-limited settings.

Paper-based microfluidic systems ( $\mu$ PADs) ${ }^{[8-17]}$ have emerged in recent years as a promising technology to address the growing need for simple, quantitative, point-of-care diagnostic devices capable of detecting different analytes from the same specimen in a single run. ${ }^{[17]}$ Paper is a useful substrate for the fabrication of microarrays through its high surface area (due to its high surface roughness and internal porosity) and high density of accessible hydroxyl functional groups. ${ }^{[18]}$ Paper is also inexpensive, flexible, easily shaped by cutting or folding, and disposable by incineration. ${ }^{[17]}$ 
To carry out separate assays simultaneously with minimal cross talk on a micro paper-based analytical device (a $\mu \mathrm{PAD}$ ), distinct microzones must vary either in terms of access to stored reagents required for the detection of each target, or in their affinity for the target molecule. The first approach has received significant attention following the development of 3D $\mu \mathrm{PADs}$ - systems which distribute the sample via vertical flow to independent test zones that store distinct reagents. ${ }^{[11,13]}$ The second approach has, so far, been largely ignored, probably due to the scarcity of methods available for assembling high quality microarrays on paper. To enable sensitive assays, these microarrays must be immobilized (preferably covalently, or, if noncovalent, with very low dissociation constants) and at high density on the surface of the substrate. ${ }^{[18,19]}$

This manuscript demonstrates the efficient synthesis of DNA oligomers 32 nucleotides in length on the surface of paper, and the fabrication of simple paper-based devices that integrate nucleic acid and protein microarrays. First, we demonstrate that the arrays of ssDNA can be used to detect fluorescently labeled DNA oligomers in solution. Next, we show that we can produce microarrays of proteins and antibodies that are two orders of magnitude more dense (based on projected footprint) than microarrays fabricated by pin-spotting. ${ }^{[4]}$ We transformed the paper-anchored ssDNA arrays into antibody microarrays via complementary-strand hybridization with ssDNA-conjugated antibodies, and show that microarrays of capture antibodies can be used to increase the sensitivity of paper devices. Last, we show that we can use paper-anchored ssDNA arrays with different sequences to assemble paper-based devices capable of detecting DNA and antibodies in the same device. 
The technique we used to fabricate microarrays takes advantage of the ease with which the surface of paper can be modified to synthesize oligomers of single-stranded DNA (ssDNA) directly. The synthesis of DNA on unmodified paper eliminates potentially time-consuming and costly purification procedures, and simplifies downstream processing. To expand the potential impact of these arrays in low-cost, point-of-care clinical diagnostics, we transformed the DNA microarrays into protein microarrays in situ using a technique developed by Jiang and Heath ${ }^{[20-23]}$ for use on the surface of silicon, glass, PDMS and other synthetic polymers. ${ }^{[24-31]}$. The technique uses an antibody that is chemically linked to ssDNA and is complementary to a surface-bound ssDNA; the antibody is immobilized on the surface via sequence-specific hybridization. DNA-directed immobilization reduces protein denaturation and enables greater orientational freedom of the antigen-binding sites than either covalent immobilization or non-specific adsorption, and yields a larger proportion of immobilized proteins (antibodies or antigens) that have unhindered binding domains. ${ }^{[25,32]}$ Other advantages of DNA-directed immobilization include increased homogeneity and reproducibility, and the consumption of less antibody per experiment. ${ }^{[32]}$ 


\section{Results and Discussion}

\section{Direct Synthesis of Single-stranded Oligonucleotides using Paper as the Solid}

\section{Support.}

The high density of hydroxyl groups on the surface of cellulose paper makes it a useful substrate for the chemistry of phosphoramidite synthesis of ssDNA. ${ }^{[33,34]}$ Whatman chromatography paper has a uniform structure and is free of coatings or binders that could interfere with the synthesis process. Figure 1 outlines the procedure used for the solid-phase synthesis of DNA carried out using paper as solid support. The post-synthesis deprotection is carried out in situ, and the process does not require purification steps.

Current methods for fabrication of DNA microarrays require synthesis of DNA onbead, deprotection and cleavage from the bead, purification by HPLC, and spotting on substrate. The low cost of the paper support $\left(\$ 0.0007 / \mathrm{cm}^{2}\right.$ Whatman Chr 1 chromatography paper), and the lack of pre- or post-synthesis steps required to activate the substrate or purify the products make the process simple and cost-effective; we believe that this method is compatible with processing on a large scale.

The high surface roughness of the paper, and its porosity, increase the area accessible to reagents and allow for larger numbers of oligonucleotides to be synthesized per area (calculated based on its planar projected footprint) than on a flat substrate (e.g. glass or polymer) with the same surface chemistry. On other substrates, increasing surface area (by applying acrylamide gels to glass slides, for example) to allow the immobilization of larger amounts of DNA resulted in greater signal intensities and an increased dynamic range. ${ }^{[35]}$ We took advantage of the high surface area of the paper by using a paper with 
a relatively high profile root mean square roughness parameter, $R_{\text {R.M.S. }}$ of $6.4 \pm 1.9 \mu \mathrm{m}$, area root mean square roughness parameter $S_{\text {R.M.S., of }} 10.7 \pm 0.6 \mu \mathrm{m}$, and the porosity, or the volume fraction of void, of $\sim 68 \% .{ }^{[36]}$ While the real surface area is usually proportional to the surface roughness, the real surface area of a rough surface is challenging to quantify, ${ }^{[37]}$ since the measurements used in fluorescent and colorimetric assays refer to intensity per two-dimensional area, we quantified the surface density of nucleotides in terms of nucleotides per projected (also referred to as plane, flat, or apparent) area of paper.

The terminal DMT protective group of the oligonucleotide, if not cleaved at the end of the synthesis, provided a useful way of characterizing the density of the oligonucleotide on the apparent surface of paper (Figure 2). The cleavage of the terminal DMT under acidic conditions can be monitored at $495 \mathrm{~nm}$ by UV spectrometer. ${ }^{[38]}$ The surface density, $\rho_{\mathrm{SP}}$ (defined as the number of molecules of oligomer per $\mathrm{cm}^{2}$ of projected area $S_{p}$ ) can be obtained from the UV absorbance at $495 \mathrm{~nm}, \mathrm{~A}_{495}$, using Eq 1, where $\varepsilon$ is the molar absorptivity of DMT, $\mathrm{v}$ is the volume of the solution in which the DMT is cleaved, $d$ is the optical path length, and $\mathrm{N}_{\mathrm{A}}$ is Avogadro's number.

$$
\rho_{S P}=\frac{\mathrm{A}_{495 \mathrm{~nm}} \times v \times N_{A}}{\varepsilon \times S_{P} \times d}
$$

Using Eq. 1, for $\varepsilon$ of DMT $7.2 \times 10^{4} \mathrm{~L} \mathrm{~mol}^{-1} \mathrm{~cm}^{-1},{ }^{[38]}$ we estimated the density of oligonucleotides per projected area of paper to be $4.5 \pm 0.5 \times 10^{14} \mathrm{~cm}^{-2}$. This value is two orders of magnitude higher than that of the current standards for DNA immobilization on 2-D substrates (for example, using pin spotting or adsorption on poly-1-lysine coated glass slides). ${ }^{[4]}$ With the exception of the first five coupling cycles, the UV absorbance at $495 \mathrm{~nm}$ of the DMT cleavage solution has plateaued, indicating that the phosphoramidite 
coupling and deprotection reactions occurred efficiently during the synthesis. The lower coupling efficiency observed in the first five coupling steps can be attributed to sluggish reactions of the DNA phosphoramidites to the less-accessible hydroxyl groups buried in the paper fibers. Since extending reaction time of the initial coupling rounds did not improve the coupling yields, we introduced spacer nucleotides of ten nucleotides at the 3 ' end of the directly synthesized oligonucleotide on paper and designed the probe-binding region to be located at the 5 'end.

In order to confirm the identity of the full-length DNA oligonucleotide generated by synthesis on paper and measure the yield, we introduced a cleavable disulfide bond in the sequence. After completing the synthesis, the terminal DMT group was left on for use as a HPLC handle. The paper-anchored DNA oligonucleotide was cleaved by reduction of the disulfide group with dithiothreitol (DTT), and the eluted material was subjected to HPLC. The main HPLC peaks were analyzed by Electrospray Ionization Mass Spectrometry (ESI-MS). Mass spectroscopy confirmed that the HPLC peak eluting at 30.9 min (Figure 2), with a mass of $6217.9 \mathrm{Da}$, was within 1.0 Da of the expected mass (with ${ }^{13} \mathrm{C}$ isotope correction), 6217.1 Da, of the full-length product after cleavage. The yield of the desired full-length oligonucleotide was estimated using absorbance data at $260 \mathrm{~nm}$, as the ratio of the area of the peak corresponding to the full-length oligonucleotide (confirmed by mass spectrometry) and the sum of the areas of the peaks in the chromatogram corresponding to truncation and full-length products. The ratio of the amount of full-length product to the total amount of oligonucleotides cleaved and eluted from paper substrate is $32 \%$ according to HPLC. 


\section{Use of ssDNA Arrays to Detect Fluorescently-labeled Oligonucleotides.}

To minimize the effects of non-specific adsorption, we used a competitive assay to detect a fluorescently-labeled target oligonucleotide, F. A Cy5 reporter was attached to the 5' end of the target. F is also complementary to the DNA oligomer S1 synthesized directly on the surface of the paper. A paper-anchored array of an oligomer S2 (see table 1 for full sequence), with a sequence non-complementary to $\mathrm{F}$, was used as a negative control.

A short ssDNA probe, Q, was designed to be complementary to F and labeled with a dark quencher (Iowa Black RQ) at the 3' end. F and Q were mixed in a 1:9 ratio and allowed to hybridize by heating to $37^{\circ} \mathrm{C}$, and the solution was then allowed to cool to room temperature. When $\mathrm{F}$ and $\mathrm{Q}$ hybridize, the fluorophore $(\mathrm{Cy} 5)$ on $\mathrm{F}$ is brought in close proximity to the quencher (Iowa Black RQ), and the hybridized product does not fluoresce.

The ssDNA anchored on the paper surface was designed to have a higher affinity for F than the probe Q. The assay is based on the competition between the DNA oligomer anchored on the paper microzone and Q for hybridization with F; blocking and washing steps are not required because a fluorescent signal is produced only as the ssDNA synthesized on paper displaces Q (from the FQ complex) to hybridize with F.

Figure 3 shows the fluorescent signal recorded after solutions of the FQ complex in concentrations between $50 \mathrm{nM}$ and $500 \mathrm{pM}$ are added to the ssDNA arrays on the surface of paper and allowed to incubate at $37^{\circ} \mathrm{C}$ for $30 \mathrm{~min}$. We are able to detect concentrations of DNA oligomer as low as $500 \mathrm{pM}$ using paper-anchored arrays of ssDNA strands complementary to F. 
Characterization of Paper-based DNA-directed Antibody Arrays. We formed microarrays by incubating solutions of ssDNA-conjugated rabbit IgG on disks ( $3 \mathrm{~mm}$ diameter) of paper on the surface of which ssDNA with complementary sequence had been synthesized. The dsDNA (formed from the hybridization of the two ssDNA strands) anchored the $\mathrm{IgG}$ to the surface of the paper.

Disks of untreated paper were used as controls. The surface of the disks was blocked with a BSA solution in PBS, then washed with PBS, and hybridized with ssDNAconjugated rabbit IgG (100 nM in PBS). Unbound conjugates were removed by washing three times with PBST buffer.

We used a fluorescently-labeled antibody (DL549 anti-rabbit IgG) as a model analyte to test the performance of the array. DL549 anti-rabbit IgG in ten-fold dilutions (1 pM to $1 \mathrm{nM})$ in a solution of goat serum $(10 \%$ serum in PBS) was added to each paper-anchored IgG microarray disk and incubated for $30 \mathrm{~min}$.

The mean fluorescence intensity of both test and control zones was measured; Figure 4 shows the calibration data in the form of the output fluorescent signal versus the concentration of DL549 anti-rabbit IgG in the sample. The LOD is $\sim 10 \mathrm{ng} \mathrm{mL}^{-1}$ (or $\sim 67 \mathrm{pM}$ ) for the assay based on DNA arrays on paper.

\section{ELISA Using Paper-based DNA-directed Antibody Arrays.}

The ultimate goal behind developing the paper-anchored antibody array technique is to measure the levels of a clinically-relevant protein in biological fluids from humans, animals, and plants. To do so, we assembled devices using paper-anchored arrays of capture antibody in microzones, and used these devices to quantify levels of $h$ CRP spiked 
into diluted human serum using a sandwich ELISA assay. We formed these microarrays by incubating solutions of ssDNA-conjugated anti- $h$ CRP antibody on disks of paper on the surface of which ssDNA with complementary sequence had been synthesized. The dsDNA (formed from the hybridization of the two ssDNA strands) anchored the anti$h$ CRP antibody to the surface of the paper.

Diluted human serum samples were spiked with recombinant $h$ CRP at concentrations ranging from $16 \mathrm{pg} \mathrm{mL}^{-1}$ to $1000 \mathrm{pg} \mathrm{mL}^{-1}$ and applied to the microzones. We used a biotinylated anti- $h \mathrm{CRP}$ as detection antibody, and streptavidin $\mathrm{Cy} 5$ as a fluorescent probe (see the experimental section for details). Figure 5 shows the calibration curve for concentration of $h \mathrm{CRP}$ vs. fluorescence intensity. The data were fit to Hill's equation with $\mathrm{R}^{2}=0.993$. The LOD was $17 \mathrm{pg} \mathrm{mL}^{-1}(\sim 1 \mathrm{pM})$.

\section{Multiplexed Assay for Detection of Fluorescently-labeled Nucleic Acids and Proteins.}

In order to show that paper-anchored ssDNA arrays with different sequences can be used to assemble paper-based devices capable of detecting DNA and antibodies from a single sample, in the same device, we used a solution containing a mixture of two model targets, a fluorescently-labeled DNA oligomer, F, and a fluorescently-labeled antibody, antirabbit IgG antibody conjugated to DL549. Figure 6 shows a schematic of the process.

To prepare a paper-based analytical device that incorporates both ssDNA and protein microarrays, we synthesized independently, on paper, ssDNA with orthogonal sequences S1 and S2 (see Table 1) and shaped the paper strips into half-disks with 3mm diameter by cutting. The array of ssDNA with sequence $\mathrm{S} 1$ was used without further modification to 
form the device. To form the protein microarray, we incubated the half disk with ssDNA with sequence S2 synthesized on its surface with a solution of complementary ssDNAconjugated rabbit IgG; the dsDNA formed from the hybridization of the two ssDNA strands anchored the IgG to the surface of the paper. We placed the two half-disks on which ssDNA and protein microarrays were formed adjacently, in order to form a single continuous paper microzone, and assembled a device as described in Figure S2. To prevent non-specific adsorption, the surface of the paper was blocked using a solution of BSA (1\% in PBS, $\mathrm{pH} 7.6)$ for $30 \mathrm{~min}$.

The fluorescently-labeled target oligomer $\mathrm{F}$ was pre-hybridized to the quencherlabeled oligomer Q, as described in the Supporting Information. A solution containing a mixture of FQ and anti-rabbit IgG antibody conjugated to DL $549(\mathrm{Ab})$, both at $5 \mathrm{nM}$ concentration, was added to each device, incubated for $30 \mathrm{~min}$ at $37^{\circ}$ and allowed to cool to room temperature. The microzones were then washed three times with PBS, and imaged with a fluorescence scanner.

Figure 6B shows an image of a typical device, and Figure $6 \mathrm{C}$ shows the average fluorescence data obtained using seven independent devices.

\section{CONCLUSION}

This work describes a method for assembling microarrays of ssDNA and proteins on the surface of paper. The strategy is based on the synthesis of ssDNA directly on paper, with modified 2'-deoxynucleoside phosphoramidites sequentially coupled to a growing 
oligonucleotide chain that is anchored in the hydroxyl groups present on the surface of cellulose paper.

This strategy of fabricating microarrays on paper is cost effective because the crude product of the synthesis is sufficiently pure to allow us to specifically differentiate the complementary DNA strand from other sequences with minimal non-specific interactions. This synthetic efficiency allows us to avoid distinct steps of DNA synthesis, purification, and immobilization; these steps are time consuming and underlie the majority of the production costs (as a reflection of solvents and reagents).

Through hybridization with complementary strands of DNA, these microarrays can capture fluorescent-labeled DNA, DNA-conjugated protein antigen, and DNAconjugated antibodies. We demonstrate the use of these microarrays to perform a sensitive sandwich ELISA to detect human CRP (LOD $13 \mathrm{ng} \mathrm{mL}^{-1}$ ), and a multiplex assay capable of detecting DNA and antibodies in the same device. The versatility of this strategy offers new approaches to integration with simple microfluidic devices, and of expansion of the repertoire of analyses, and the sensitivity of the assays, that can be conducted using paper.

\section{ACKNOWLEDGEMENTS}

We are thankful for the financial support provided by the Bill and Melinda Gates Foundation under award 51308. A. C. G. was partially supported by DTRA award \# HDTRA1-14-C-0037. J. N. was partially supported by an Eli Lily Organic Chemistry Graduate Fellowship. F.G. acknowledges funding by German Research Foundation (DFG) under contract GU 1468/1-1. 


\section{REFERENCES}

[1] G. Walter, K. Bussow, A. Lueking, J. Glokler, Trends Mol. Med. 2002, 8, 250.

[2] B. Zhumabayeva, A. Chenchik, P. D. Siebert, M. Herrler, Adv. Biochem. Eng. Biotechnol. 2004, 86, 191.

[3] M. B. Miller, Y. W. Tang, Clin. Microbiol. Rev. 2009, 22, 611.

[4] Y. S. Shin, H. Ahmad, Q. H. Shi, H. Kim, T. A. Pascal, R. Fan, W. A. Goddard, J. R. Heath, ChemPhysChem 2010, 11, 3063.

[5] I. Barbulovic-Nad, M. Lucente, Y. Sun, M. Zhang, A. R. Wheeler, M. Bussmann, Crit. Rev. Biotechnol. 2006, 26, 237.

[6] M. Dufva, Biomol. Eng. 2005, 22, 173.

[7] S. Taylor, S. Smith, B. Windle, A. Guiseppi-Elie, Nucleic Acids Res. 2003, 31, e87.

[8] A. W. Martinez, S. T. Phillips, M. J. Butte, G. M. Whitesides, Angew. Chem. 2007, 46, 1318.

[9] A. W. Martinez, S. T. Phillips, E. Carrilho, S. W. Thomas, H. Sindi, G. M. Whitesides, Anal. Chem. 2008, 80, 3699.

[10] A. W. Martinez, S. T. Phillips, Z. Nie, C. M. Cheng, E. Carrilho, B. J. Wiley, G. M. Whitesides, Lab Chip 2010, 10, 2499.

[11] A. W. Martinez, S. T. Phillips, G. M. Whitesides, Proc. Natl. Acad. Sci. U. S. A. 2008, 105, 19606.

[12] A. W. Martinez, S. T. Phillips, G. M. Whitesides, E. Carrilho, Anal. Chem. 2010, 82,3 .

[13] H. Liu, R. M. Crooks, J. Am. Chem. Soc. 2011, 133, 17564.

[14] H. Liu, X. Li, R. M. Crooks, Anal. Chem. 2013, 85, 4263.

[15] S. J. Vella, P. Beattie, R. Cademartiri, A. Laromaine, A. W. Martinez, S. T.

Phillips, K. A. Mirica, G. M. Whitesides, Anal. Chem. 2012, 84, 2883. 
[16] S. Wang, L. Ge, X. Song, J. Yu, S. Ge, J. Huang, F. Zeng, Biosens. Bioelectron. 2012, 31, 212.

[17] A. K. Yetisen, M. S. Akram, C. R. Lowe, Lab Chip 2013, 13, 2210.

[18] J. Credou, T. Berthelot, J. Mater. Chem. 2014, 2, 4767.

[19] F. Kong, Y. F. Hu, Anal. Bioanal. Chem. 2012, 403, 7.

[20] R. C. Bailey, G. A. Kwong, C. G. Radu, O. N. Witte, J. R. Heath, J. Am. Chem. Soc. 2007, 129, 1959.

[21] C. Boozer, J. Ladd, S. Chen, S. Jiang, Anal. Chem. 2006, 78, 1515.

[22] R. Fan, O. Vermesh, A. Srivastava, B. K. H. Yen, L. D. Qin, H. Ahmad, G. A.

Kwong, C. C. Liu, J. Gould, L. Hood, J. R. Heath, Nat. Biotechnol. 2008, 26, 1373.

[23] C. Boozer, J. Ladd, S. Chen, Q. Yu, J. Homola, S. Jiang, Anal. Chem. 2004, 76, 6967.

[24] P. Angenendt, J. Glokler, J. Sobek, H. Lehrach, D. J. Cahill, J. Chromatogr. A 2003, 1009, 97.

[25] R. C. Bailey, G. A. Kwong, C. G. Radu, O. N. Witte, J. R. Heath, J. Am. Chem. Soc. 2007, 129, 1959.

[26] R. Benters, C. M. Niemeyer, D. Drutschmann, D. Blohm, D. Wohrle, Nucleic Acids Res. 2002, 30.

[27] C. Boozer, J. Ladd, S. F. Chen, S. T. Jiang, Anal. Chem. 2006, 78, 1515.

[28] E. S. Douglas, R. A. Chandra, C. R. Bertozzi, R. A. Mathies, M. B. Francis, Lab Chip 2007, 7, 1442.

[29] M. Dufva, Biomol. Eng. 2005, 22, 173.

[30] R. Fan, O. Vermesh, A. Srivastava, B. K. H. Yen, L. Qin, H. Ahmad, G. A.

Kwong, C.-C. Liu, J. Gould, L. Hood, J. R. Heath, Nat. Biotechnol. 2008, 26, 1373.

[31] H. Schroeder, M. Adler, K. Gerigk, B. Mueller-Chorus, F. Goetz, C. M.

Niemeyer, Anal. Chem. 2009, 81, 1275.

[32] R. Wacker, H. Schroder, C. M. Niemeyer, Anal. Biochem. 2004, 330, 281.

[33] S. L. Beaucage, R. P. Iyer, Tetrahedron 1992, 48, 2223.

[34] S. Agrawal, Protocols for Oligonucleotides and Analogs: Synthesis and Properties, Vol. 20, Springer, 1993.

[35] D. Proudnikov, E. Timofeev, A. Mirzabekov, Anal. Biochem. 1998, 259, 34.

[36] A. C. Glavan, R. V. Martinez, A. B. Subramaniam, H. J. Yoon, R. M. D. Nunes, H. Lange, M. M. Thuo, G. M. Whitesides., Adv. Funct. Mater. 2013 DOI: 10.1002/adfm.201300780.

[37] M. G. Donoso, A. Méndez-Vilas, J. M. Bruque, M. L. González-Martin, Int. Biodeter. Biodegr. 2007, 59, 245.

[38] ATDBIO, "Solid-Phase Oligonucleotide synthesis", retrieved at http://www.atdbio.com/content/17/Solid-phase-oligonucleotide-synthesis 2014. 